\title{
Distinct Functional States of Astrocytes During Sleep and Wakefulness: Is Norepinephrine the Master Regulator?
}

\author{
John O'Donnell • Fengfei Ding • Maiken Nedergaard
}

Published online: 29 January 2015

(C) Springer International Publishing AG 2015

\begin{abstract}
Astrocytes are the chief supportive cells in the central nervous system, but work over the past 20 years have documented that astrocytes also contribute to complex neural processes, such as working memory. Recent discoveries of norepinephrine-mediated astrocytic $\mathrm{Ca}^{2+}$ responses have raised the possibility that astrocytic activity in the adult brain is driven by global responses to changes in behavioral state. Moreover, analysis of the interstitial space volume suggests that astrocytes may undergo changes in cell volume in response to activation of norepinephrine receptors. This review will focus on what is known about astrocytic functions within the nervous system and how these functions interrelate with rapid changes in behavioral state mediated by norepinephrine signaling.
\end{abstract}

Keywords Astrocytes · Norepinephrine - Sleep · Circadian · Homeostasis $\cdot$ State-dependent $\cdot$ Wakefulness $\cdot$ Potassium . Calcium $\cdot$ Behavioral state

\section{Introduction}

Each day the nervous system is continually confronted with a diverse set of challenges. On short timescales, ongoing behavioral demands drive rapid changes in network connectivity, attention, and sensory processing. Over longer timeframes,

This article is part of the Topical Collection on Function of Sleep

J. O’Donnell $(\varangle) \cdot$ F. Ding $\cdot$ M. Nedergaard

Center for Translational Neuromedicine, University of Rochester

Medical School, Rochester, NY 14642, USA

e-mail: John_ODonnell@urmc.rochester.edu

M. Nedergaard $(\square)$

Division of Glial Disease and Therapeutics, Center for Translational

Neuromedicine, University of Rochester Medical Center,

601 Elmwood Ave, Rochester, NY 14642, USA

e-mail: nedergaard@urmc.rochester.edu daily rhythms in activity and wakefulness permit the formation, cross-sampling, and consolidation of memories. Coupled to this capacity to flexibly alter state to respond to behavioral demands is the need to maintain homeostasis, to reign in excesses in activity and metabolism, and to provide mechanisms to reset networks to perform optimally over extended periods of times. The state shifts necessary to perform optimally throughout life arise from the balance of these metabolic, plastic, and behavioral demands. Driven by alterations in neuromodulator release, both local and global changes can drive the brain toward activity or recovery. These rapid shifts permit animals to respond adaptively to changing behavioral demands, permitting the brain to forego sleep and other restorative states and driving the brain into higher vigilance states. On the converse, the accumulated pressure of long periods of vigilance acts to push longer periods of recovery sleep. How the brain regulates the balance of these states and the mechanisms underlying them remains a mystery. This review will address the most fundamental of these state shifts, the shift between sleep to awake states, from the perspective of the principal homeostatic cells in the brain, astrocytes.

This cycling between more and less active states arises both from innate needs to restore homeostasis as well as the need to override these signals when necessary. At the core of this shift are astrocytes. To begin understanding how astrocytes may be involved in state shifts, this review will first explore the wellestablished supportive role of astrocytes. How these functions interrelate with complex homeostatic forces arising from both innate circadian cycling and activity-dependent pressures will then be addressed. While the brain acts through these rhythmic pressures to oscillate between sleep and awake states, the regulation of how the nervous system is capable of altering these drives in response to external behavioral pressures is far more complex.

Integrating these internal and external pressures and providing a mechanism for rapid state shifts are many complementary neuromodulatory systems that are capable of altering network activity over broad areas of cortex. As astrocytes have been 
shown to play an active role in regulating neuronal activity, and as recent work has demonstrated that astrocytic activity is restricted to awake states and requires noradrenergic signaling $[1,2]$, they may play a substantial role in the regulation of these state-dependent shifts in network activity. Through neuromodulation and astrocytic activity, the brain is capable of rapid adaptation to immediate demands. Beyond balancing homeostatic needs and the need to rapidly respond, sleep, neuromodulation, and astrocytic activity are at the core of how the nervous system is able to form and consolidate new memories. To understand this, this review will focus on recent work demonstrating a strong link between synaptic plasticity and metabolic demands, with plasticity being sculpted by neuromodulation and astrocytic activity.

At the center of all these processes is the idea that the brain must be able to balance the need for periods of restorative functions with the need to respond rapidly to changing environments. Through this, the brain is able to maintain longterm homeostasis while integrating new memories to permit long-term behavioral adaptation, and as both the principle regulators of homeostasis and as targets of neuromodulatory systems, astrocytes are poised to play a substantial role in balancing both these essential functions.

\section{Astrocytes: Gatekeepers of Homeostasis}

The first question in understanding how sleep-to-awake shifts occur is what roles astrocytes play as homeostatic regulators in the brain. In the brain, astrocytes form a fine network of processes that both completely cover the endothelial tube and associated pericytes [3] as well as covering tens of thousands of synapses in the human brain [4] (Fig. 1). Though connected via gap junctions with neighboring astrocytes, in the healthy brain, individual astrocytes tend to form nonoverlapping domains, placing them at the center of regulating local homeostatic functions [4]. Through these domains, astrocytes are capable of regulating a host of processes, including ionic homeostasis, neurovascular communication and metabolite uptake, and neurotransmitter clearance.

Beginning with discoveries that astrocytes are highly permeable to [5] and rapidly uptake $\mathrm{K}^{+}$[6], the realization that astrocytes may play a strong role in homeostasis began to emerge. At a basic level, astrocytes maintain local fluid and ionic homeostasis. In response to hormonal changes $[7,8]$ and through translocation with transmitters, astrocytes alter their regulation of chloride and water uptake. During periods of high neuronal activity, astrocytes increase $\mathrm{K}^{+}$uptake [9], preventing extracellular accumulation and consequent neuronal depolarization (Fig. 1). In pathological states, dysregulation of $\mathrm{K}^{+}$homeostasis is known to drive seizures [10], and changes in resistance to water flow through decreased expression of the astrocytic water channel aquaporin-4 (AQP4) are known to alter post-injury edema [11]. In this way, astrocytes regulate the volume of their surrounding tissue, while providing a means to support neurons through buffering of excitatory ions.

Astrocytes also act as principle intermediaries between neuronal activity and vascular responses. With their vascular processes ensheathing the vascular wall, astrocytes are the principle cells exposed to signaling molecules carried by the general circulation as well as metabolic substrates [12]. The presence of astrocytes at the blood-brain barrier enhances tight-junction formation [12]. Astrocytes also organize the polarized network of transport molecules that regulate the influx of molecules into the nervous system [12]. Opposite, pathological disruption leading to reactive gliosis lead to neuronal cell loss and neurological disorders [13]. The position of astrocytes as a bridge between neurons and the vasculature further allows astrocytes to provide energy substrates for neurons [14] as well as to drive vasodilation in response to sensory input [15]. Beyond acting as conduits for metabolic substrates, astrocytes are the sole source of glycogen in the brain. This permits them to function as metabolic buffers. Similarly to ionic homeostasis, these functions act both to provide support during normal periods while acting as buffers during periods of high-metabolic demand. Whether actively supplying neurons with metabolic substrates such as glucose and lactate, or driving increases in blood flow following spikes in neuronal activity, astrocytes act as intermediaries between the metabolic demands of the brain and the current metabolic state of the body as a whole.

Augmenting these broader roles of astrocytes in the regulation of metabolic and ionic homeostasis, astrocytes also play a significant role in the uptake of neurotransmitters. Through the uptake of glutamate by the transporters GLT1 and GLAST, astrocytes rapidly clear synapses of excess glutamate, preventing excessive neuronal activity and enhancing synaptic efficacy [16-18]. Additionally, through regulation of GABA uptake, astrocytes play a role in maintaining inhibitory tone within the brain $[19,20]$.

Together, these key functions place astrocytes at the center of maintaining brain homeostasis. By ensheathing both synapses and the vascular wall, astrocytes prevent excesses in activity while also placing them at the center of communicating activity-dependent changes in metabolic demand from neurons to the vasculature. This allows astrocytes to maintain homeostasis, buffering increases in neuronal activity and integrally supporting a functioning nervous system.

\section{Cleaning House}

The drive for sleep is one of the clearest reflections of the nervous system's need to maintain homeostasis. It is clear that long periods of wakefulness drive increases in the need for 


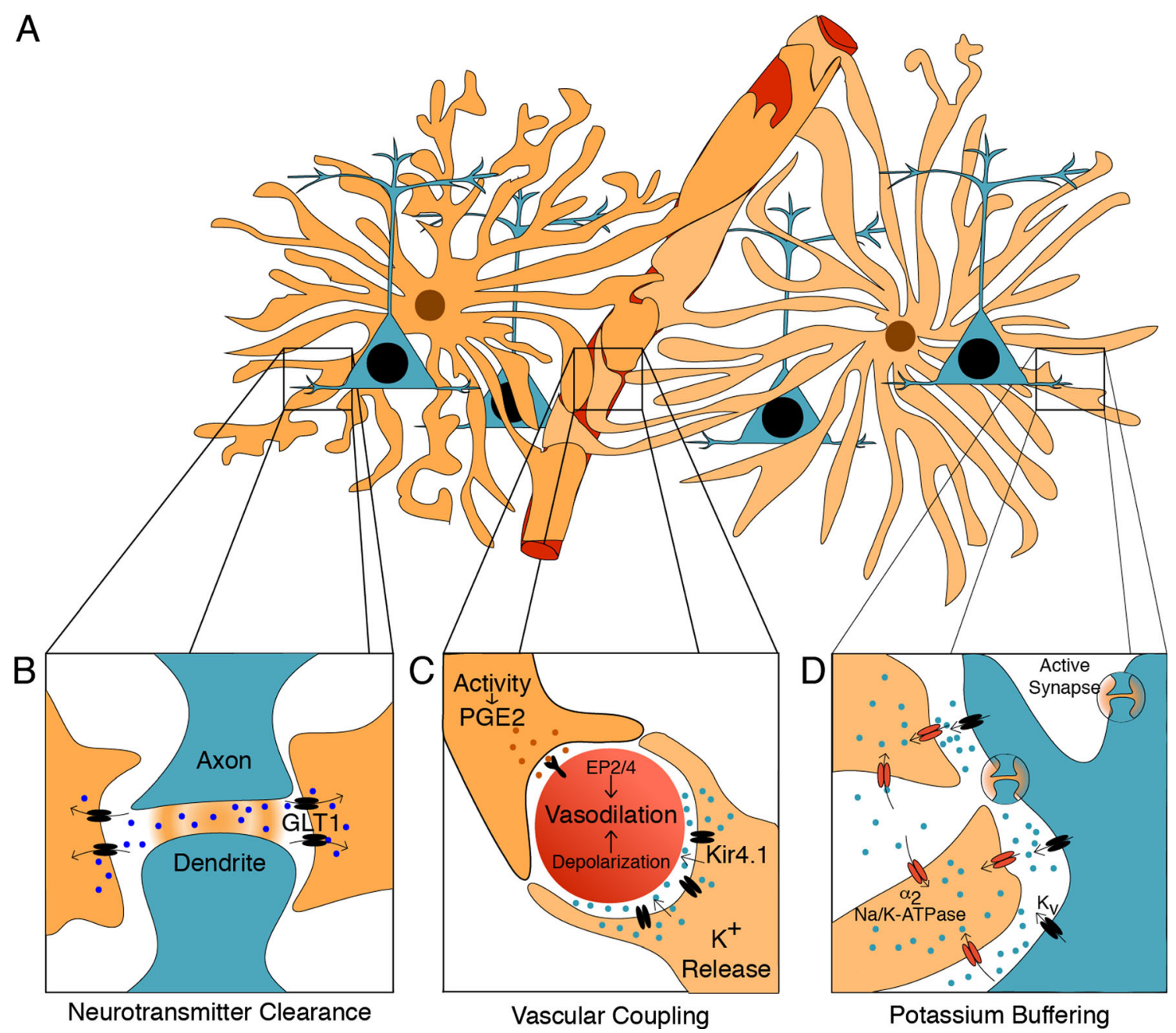

Fig. 1 Astrocytes are critical support cells of the brain. a A schematic drawing of astrocytic complexity and coverage of brain vasculature. b Astrocytes act as the predominate route for clearance of excitatory extracellular glutamate in the nervous system [16]. c In response to heightened neuronal activity or reduced oxygen tension, astrocytes release PGE2 [68] and potassium [69], stimulating vasodilation. d During neuronal activity, astrocytes' rapidly uptake $\mathrm{K}^{+}$to maintain homeostasis and prevent excessive neuronal depolarization [9] sleep, but the reasons sleep is required remain poorly understood. Many different theories have been proposed, including increased synaptic potentiation throughout the day [21•], the accumulation of metabolites or other signals of "sleep-pressure, " or simply the innate need to synchronize activity with the time of day. Uniting these theories is the common theme that sleep acts to maintain metabolic homeostasis, with periods of high activity depleting energy stores and leading to the formation of toxic metabolites that hinder further activity. Through their roles in regulating homeostasis, astrocytes are critical to maintaining this balance and driving recovery during sleep.

Periods of high activity also lead to the accumulation of toxic metabolites within the brain. While it had been longestablished that, unlike the blood-brain barrier, the brain-CSF barrier permits the passage of relatively large molecules [22, 23], with bulk flow of interstitial fluid occurring in the brain $[24,25]$, in a recent study this flow was found to be dependent upon astrocytes [26]. This discovery has opened up numerous possibilities for understanding the regulation of metabolite clearance in the brain. Whereas simple diffusion could not effectively explain movement of solutes over long distances through the highly convoluted interstitial space of the brain, convective fluid flow, termed the glymphatic system, has now been found to drive both the influx and clearance of molecules.

Critical in regulating this system is the expression of the astrocytic water channel, AQP4. Genetic deletion of this channel, generally localized to the perivascular endfeet of astrocytes [27], has been found to substantially reduce the influx and clearance of tracers injected into either the CSF or brain interstitial space [26]. Moreover, further studies have shown that loss of AQP4 targeting to the perivascular zone is associated with substantial dysfunction in bulk fluid flow in Alzheimer disease [26], traumatic brain injury [28], and aging [29]. This necessity for astrocytic AQP4 regulation of bulk fluid flow underlies the need for coherent astroglial activity in regulating glymphatic activity. The magnitude of this 
glymphatic activity was found to be regulated by sleep/wake cycles in the mouse, with significantly more tracer influx into the brain during both true sleep and anesthetized states relative to the awake state [30]. This finding, coupled with the discovery that these changes in flux relate to substantial $(>50 \%)$ changes in the total volume of brain extracellular space between sleep and awake states places glymphatic activity at the center of the homeostatic drive for sleep, permitting the clearance of toxic metabolites including amyloid- $\beta[30]$.

Though the specific mechanisms underlying this shift in extracellular space and enhanced glymphatic flux through the brain during sleep are yet to be elucidated, this system suggests a novel mechanism in how sleep may act to rejuvenate the mind. This finding is augmented by the further discovery that glymphatic clearance is dependent on the widespread activity of noradrenergic receptors, major regulators of sleep and awake states. This synchrony strongly suggests the involvement of astrocytes in maintaining homeostasis both in the stark shift from sleep to awake and perhaps also in other behavioral states $[31 \bullet, 32 \bullet]$. In this, it appears that astrocytes coordinate the cortex to balance behavioral needs with longterm homeostasis in response to environmental demands.

\section{Shifting States: Neuromodulators}

While the above has focused on the need for sleep to restore homeostasis and the potential roles for astrocytic activity within this system, the capacity of the brain to balance accumulated sleep pressure and the need for long-term homeostasis with shifting behavioral priorities require the overarching control of neuromodulatory systems. Over the past several decades, extensive literature has identified a diverse array of neuromodulators in regulating sleep and different behavioral states, including norepinephrine, serotonin, dopamine, acetylcholine, histamine, orexin (hypocretin), and others [33]. While each of these transmitters plays a distinct role in driving wakefulness, and disruption to individual systems can cause compensatory changes in others to maintain normal function, norepinephrine's (NE) known role in altering sleep/wake cycles [34], glymphatic activity [30], astrocytic responses [1, 2], and plasticity make it of particular interest for understanding the potential role of astrocytes in responding to changes in behavioral state.

$\mathrm{NE}$ is involved in diverse processes from driving wakefulness, resetting networks to rapidly attend to sudden unexpected events, and laying the foundation for plasticity and longterm behavioral adaptation. Cortical NE is released by projections from the locus coeruleus (LC), a small nucleus in the pons that consists of only 20-50,000 neurons in the human brain [35]. LC release is achieved largely via non-junctional varicosities, or en passant synapses, with as few as $10 \%$ of release sites being associated with traditional synaptic contacts
[36]. This arrangement facilitates the ability of NE to drive changes across broad swathes of cortex and multiple cell types through volume transmission. Firing of the $\mathrm{LC}$ is associated both with changes in basal excitatory tone as well as global responses to sudden environmental challenges and behaviorally relevant stimuli [37]. To accomplish this, the LC engages in two distinct modes of firing: tonic firing $(0.1-5 \mathrm{~Hz})$, which drives wakefulness and arousal, and phasic bursts of two to three action potentials $(10-20 \mathrm{~Hz})$, which occurs in response to top-down attentional modulation as well as novel, salient, or polymodal sensory stimuli [38]. Through these two firing modes, the LC is able to maintain attentional circuits needed for working memory and unimodal sensory processing, while facilitating shifts in connectivity and narrow enhancement of strong stimuli needed to encode important behavioral stimuli and integrate polymodal sensory information [38-40].

This ability to rapidly alter large areas of cortex in response to behavioral demands allows NE to exert a profound effect on sleep/wake cycles and shifts in attention. By (1) playing a permissive role in astrocytic $\mathrm{Ca}^{2+}$ responses $[1,2]$, thereby driving their ability to optimize responses to changing metabolic and neuronal processing demands, (2) driving broad depolarizing shifts in neuronal membrane potential to facilitate awake states [41], and (3) reducing the extracellular space in awake states [30], NE is an exemplar of how neuromodulation synchronizes cortical responses through a multitude of different behavioral states. By varying their activity, neuromodulators permit brain responses to a variety of demands, and it is this flexibility that underlies how the brain is able to balance the need for the regenerative effects of sleep with the need to be able to respond when important events preclude spending several hours unconscious.

\section{Opening the Gates: Astrocytes as Active Regulators of Neuronal Activity}

While the supporting roles above demonstrate the necessity for astrocytes in maintaining homeostasis, these functions do not act as simple passive mechanisms for responding to neuronal activity. For the past two decades, research has elucidated a myriad of changes in these support functions following release of neurotransmitters, neuromodulators, hormones, and many other signaling molecules. From these studies, it has become clear that, far from acting as simple support cells, astrocytes are capable of readily acting to accelerate or brake network activity in response to changes in their environment.

Beginning with work done in the early 1990s, it was realized that, though electrically non-excitable, astrocytes respond to a wide array of transmitters, signaling molecules, and changes in their environment with large spikes in intracellular calcium. While still a matter of debate as to what the exact function of these responses are, at their broadest they have 
been shown to drive changes in neuronal activity. Many of these responses are distinctly related to the support functions described earlier including, among others, increased vasodilation $[42,43]$ and inhibition of neuronal activity through increased $\mathrm{K}^{+}$uptake [44]. Notably however, it has been the idea that astrocytes themselves may be actively releasing signaling molecules through a variety of mechanisms including hemichannels [45], transporters [46], volume-activated channels [47], and exocytosis [48]. With early work finding that astrocytes respond to the local signaling molecule glutamate, many studies have focused on the potential for astrocytes to release signaling molecules in direct response to local activity, as part of a "tripartite synapse." In this model, astrocytes are thought to have the potential to release putative "gliotransmitters" such as glutamate [49, 50], ATP [51-53], D-serine [54], PGE2 [55], and GABA [56] to directly modulate local synaptic activity. However, though a multitude of studies have focused on astrocytic exocytosis of transmitter molecules, the capacity for astrocytes to release transmitter from intracellular vesicles has been a matter of debate. In many of these studies, the potential for astrocytic modulation of synaptic activity through exocytosis was explored using a mouse-line expressing a dominant-negative form of the SNARE protein under the astrocyte-specific GFAP promoter to prevent vesicle binding. However, with a recent study demonstrating the expression of this protein within neuronal cell populations as well as substantially reduced cortical activity in these mice, its efficacy as a model for astrocytic exocytosis of transmitters is questionable [57]. In addition, while many of the preceding studies used preparations from young, immature mice, recent studies in adult mice have failed to demonstrate astrocytic $\mathrm{Ca}^{2+}$ responses to glutamate [58], raising the possibility that earlier work reflected a developmental phenomenon. In light of this work, the question of whether astrocytes respond directly to individual synapses and local activity remains open to debate.

Challenging the idea that astrocytes respond predominately to local transmitters, two recent papers have shown that astrocytic $\mathrm{Ca}^{2+}$ increases in awake, behaving mice is dependent on the widespread release of NE throughout the cortex $[1,2]$. In responding to $\mathrm{NE}$ through the $\alpha_{1}$-adrenergic receptor ( $\alpha_{1}$-AR) following startle stimulation and/or movement, astrocytic $\mathrm{Ca}^{2+}$ increases appear to be driven by global changes in state rather than local neuronal activity. Though these papers do not exclude the possibility of modulation of $\mathrm{Ca}^{2+}$ responses through local signals and other cues, they do demonstrate that these responses can be prevented through either blockade of the $\alpha_{1}$-AR or pharmacological ablation of the source of cortical NE, the locus coeruleus (LC). This has further been corroborated with the finding that astrocytic $\mathrm{Ca}^{2+}$ responses are blunted or absent in the presence of anesthesia-a state of extremely low LC activity [42, 59]. Together, these papers suggest that astrocytes tend to respond in synchrony across large volumes of cortex in parallel with broad neuromodulatory inputs from the LC. These global responses may alter synaptic efficacy, set inhibitory tone, or alter vascular dynamics to set the tonus for neuronal excitability and coordinate rapid, widespread changes in state.

Over the last 20 years, a huge variety of astrocytic responses have been uncovered that may play a role in regulating local neuronal activity. However, many of these responses have been found only in immature animals, with the breadth of these responses indicating that astrocytes are acting to complement neuronal activity. Astrocytes have been shown to drive a wide range of effects including direct neuronal modulation by transmitters, inhibition through altered ion homeostasis, metabolite release, and vascular coupling. While some of these functions likely arise from local changes in activity, the finding that astrocytes undergo widespread $\mathrm{Ca}^{2+}$ waves in response to bursts of locus coeruleus' activity suggest that they play a broader role in facilitating rapid responses during startle and locomotion. This raises the intriguing possibility that astrocytes act not just as local homeostats but as central integrators for the convergence of local activity and global changes resulting from shifting behavioral demands.

\section{Sleep and Plasticity}

At the convergence of neuromodulators' role in driving shifts in behavioral state is the question of learning and plasticity. Beyond its role in simply maintaining metabolic homeostasis, sleep is necessary for the long-term consolidation of new memories [60]. From a broad perspective, this function can be understood as an overarching need for different information processing states between initial encoding of memories during wake states and extensive comparisons during sleep necessary for long-term consolidation. These different processing modes coincide with changes in neuromodulation, with neuromodulators being necessary for encoding the salience of events in order to drive long-term potentiation of synapses [61, 62]. In this way, the necessity of sleep extends beyond homeostasis to far more complex needs of the nervous system.

Of particular interest in plasticity is the concept of the synaptic homeostasis hypothesis $[21 \bullet, 63]$. In this hypothesis, it has been shown that throughout waking periods there is a consistent increase in evoked activity in the brain, correlating to an overall increase in synaptic potentiation [64]. Conversely, following slow-wave sleep, there is a consistent decrease in overall evoked activity, reflecting a down-scaling in the expression of synaptic proteins. Importantly, $20 \%$ of plasticityrelated genes that increase during wakefulness are under control of NE [65, 66]. This wake/sleep balance may reflect differences in how memories are encoded and consolidated during different behavioral states. 
These findings illustrate the complex interrelationship between the need for sleep to permit functions as intricate as memory consolidation and behavioral adaptation as well as more basic functions such as metabolic homeostasis. With increased metabolic demands arising from long periods of activity, and with potential ceiling effects arising from net synaptic potentiation during these periods, the shift to sleep permits the brain to restore a baseline level of activity in order to achieve long-term homeostasis and permit future learning. Crucially, with the involvement of neuromodulation in LTP as well as in regulating behavioral state, these findings suggest an overarching continuum of behavioral states that are adapted to balance the effects of sustained activity with current behavioral priorities. Moreover, with astrocytes' involvement in noradrenergic responses in sleep/wake cycles as well as with the necessity of their responses to NE in permitting LTP and memory consolidation through metabolic support [67], these findings suggest that astrocytes play a role in regulating responses to neuromodulation based off of current metabolic demands in their role as territorial integrators and gatekeepers of homeostasis.

\section{Conclusion}

The capacity of the brain to integrate the innate need for regenerative periods with the ability to flexibly alter cognitive state in response to external demands is critical to the survival of an organism. This balance is maintained by both local metabolic demands and global regulation by neuromodulators. Deeply involved in this are astrocytes. Regulated by NE, these cells buffer metabolic activity, regulate interstitial fluid fluxes, and alter neuronal activity. This integration of local metabolic demand with widespread shifts resulting from NE release places astrocytes in an ideal position to alter how the brain functions under a variety of behavioral conditions. Though the exact mechanisms are just beginning to be unraveled, this convergence of neuromodulation with basic homeostatic demands raises several intriguing possibilities. First, changes in the extracellular space and glymphatic clearance of metabolites during sleep may promote plasticity by decreasing synaptic efficacy and allowing greater activation of extrasynaptic receptors through volume transmission. Second, the multiple roles of neuromodulators in driving both responses to salient stimuli as well as most basal wakefulness suggests that these are tightly interrelated phenomena. Finally, the substantial changes between sleep and awake states suggest that astrocytic activity is tightly regulated by neuromodulatory systems, and that this activity may be altered depending on the specific state an organism is in. In this way, astrocytic functions can be viewed not only as a single or dual state of activity for supporting and integrating local neuronal activity, but rather as existing in a continuum of states under the flexible control of NE to permit a myriad of responses.

\section{Compliance with Ethics Guidelines}

Conflict of Interest John O'Donnell, Fengfei Ding, and Maiken Nedergaard have received grants from the National Institutes of Health (NIH).

Human and Animal Rights and Informed Consent This article does not contain any studies with human or animal subjects performed by any of the authors.

\section{References}

Papers of particular interest, published recently, have been highlighted as:

- Of importance

1. Ding F, O’Donnell J, Thrane AS, Zeppenfeld D, Kang H, Xie L, et al. alpha1-Adrenergic receptors mediate coordinated $\mathrm{Ca}^{2+}$ signaling of cortical astrocytes in awake, behaving mice. Cell Calcium. 2013;54:387-94.

2. Paukert M, Agarwal A, Cha J, Doze VA, Kang JU, Bergles DE. Norepinephrine controls astroglial responsiveness to local circuit activity. Neuron. 2014;82:1263-70.

3. Mathiisen TM, Lehre KP, Danbolt NC, Ottersen OP. The perivascular astroglial sheath provides a complete covering of the brain microvessels: an electron microscopic $3 \mathrm{D}$ reconstruction. Glia. 2010;58:1094-103.

4. Oberheim NA, Takano T, Han X, He W, Lin JH, Wang F, et al. Uniquely hominid features of adult human astrocytes. J Neurosci. 2009;29:3276-87.

5. Orkand RK, Nicholls JG, Kuffler SW. Effect of nerve impulses on the membrane potential of glial cells in the central nervous system of amphibia. J Neurophysiol. 1966;29:788-806.

6. Hertz L. Possible role of neuroglia: a potassium-mediated neuronal-neuroglial-neuronal impulse transmission system. Nature. 1965;206:1091-4.

7. Katay L, Latzkovits L, Fonagy A, Janka Z, Lajtha A. Effects of arginine vasopressin and atriopeptin on chloride uptake in cultured astroglia. Neurochem Res. 1998;23:831-6.

8. Hertz L, Chen Y, Spatz M. Involvement of non-neuronal brain cells in AVP-mediated regulation of water space at the cellular, organ, and whole-body level. J Neurosci Res. 2000;62:480-90.

9. Walz W. Role of astrocytes in the clearance of excess extracellular potassium. Neurochem Int. 2000;36:291-300.

10. Rangroo Thrane V, Thrane AS, Wang F, Cotrina ML, Smith NA, Chen $\mathrm{M}$, et al. Ammonia triggers neuronal disinhibition and seizures by impairing astrocyte potassium buffering. Nat Med. 2013;19:1643-8.

11. Manley GT, Fujimura M, Ma T, Noshita N, Filiz F, Bollen AW, et al. Aquaporin-4 deletion in mice reduces brain edema after acute water intoxication and ischemic stroke. Nat Med. 2000;6:159-63.

12. Abbott NJ, Ronnback L, Hansson E. Astrocyte-endothelial interactions at the blood-brain barrier. Nat Rev Neurosci. 2006;7:41-53.

13. Wang M, Iliff JJ, Liao Y, Chen MJ, Shinseki MS, Venkataraman A, et al. Cognitive deficits and delayed neuronal loss in a mouse model of multiple microinfarcts. J Neurosci. 2012;32:17948-60. 
14. Chih CP, Roberts Jr EL. Energy substrates for neurons during neural activity: a critical review of the astrocyteneuron lactate shuttle hypothesis. J Cereb Blood Flow Metab. 2003;23:1263-81.

15. Gordon GR, Choi HB, Rungta RL, Ellis-Davies GC, MacVicar BA. Brain metabolism dictates the polarity of astrocyte control over arterioles. Nature. 2008;456:745-9.

16. Danbolt NC. Glutamate uptake. Prog Neurobiol. 2001;65:1-105.

17. Rothstein JD, Martin L, Levey AI, Dykes-Hoberg M, Jin L, Wu D, et al. Localization of neuronal and glial glutamate transporters. Neuron. 1994;13:713-25.

18. Oliet SH, Piet R, Poulain DA. Control of glutamate clearance and synaptic efficacy by glial coverage of neurons. Science. 2001;292: 923-6.

19. Shigetomi E, Tong X, Kwan KY, Corey DP, Khakh BS. TRPA1 channels regulate astrocyte resting calcium and inhibitory synapse efficacy through GAT-3. Nat Neurosci. 2012;15:70-80.

20. Yoon BE, Woo J, Chun YE, Chun H, Jo S, Bae JY, et al. Glial GABA, synthesized by monoamine oxidase B, mediates tonic inhibition. J Physiol. 2014;592:4951-68.

21. Tononi G, Cirelli C. Sleep and the price of plasticity: from synaptic and cellular homeostasis to memory consolidation and integration. Neuron. 2014;81:12-34. Excellent recent review on synaptic scaling and plasticity in sleep and awake states.

22. Rennels ML, Blaumanis OR, Grady PA. Rapid solute transport throughout the brain via paravascular fluid pathways. Adv Neurol. 1990;52:431-9.

23. Rennels ML, Gregory TF, Blaumanis OR, Fujimoto K, Grady PA. Evidence for a 'paravascular' fluid circulation in the mammalian central nervous system, provided by the rapid distribution of tracer protein throughout the brain from the subarachnoid space. Brain Res. 1985;326:47-63.

24. Abbott NJ. Evidence for bulk flow of brain interstitial fluid: significance for physiology and pathology. Neurochem Int. 2004;45:545-52.

25. Cserr HF, Ostrach LH. Bulk flow of interstitial fluid after intracranial injection of blue dextran 2000. Exp Neurol. 1974;45:50-60.

26. Iliff JJ, Wang M, Liao Y, Plogg BA, Peng W, Gundersen GA, et al. A paravascular pathway facilitates CSF flow through the brain parenchyma and the clearance of interstitial solutes, including amyloid beta. Sci Transl Med. 2012;4:147ra111.

27. Neely JD, Amiry-Moghaddam M, Ottersen OP, Froehner SC, Agre P, Adams ME. Syntrophin-dependent expression and localization of aquaporin-4 water channel protein. Proc Natl Acad Sci U S A. 2001;98:14108-13.

28. Ren Z, Iliff JJ, Yang L, Yang J, Chen X, Chen MJ, et al. 'Hit \& Run' model of closed-skull traumatic brain injury (TBI) reveals complex patterns of post-traumatic AQP4 dysregulation. J Cereb Blood Flow Metab. 2013;33:834-45.

29. Kress BT, Iliff JJ, Xia M, Wang M, Wei HS, Zeppenfeld D, et al. Impairment of paravascular clearance pathways in the aging brain. Ann Neurol. 2014;76:845-61.

30. Xie L, Kang H, Xu Q, Chen MJ, Liao Y, Thiyagarajan M, et al. Sleep drives metabolite clearance from the adult brain. Science. 2013;342:373-7.

31. Polack PO, Friedman J, Golshani P. Cellular mechanisms of brain state-dependent gain modulation in visual cortex. Nat Neurosci. 2013;16:1331-9. Demonstrates NE-dependent depolarization of V1 neurons during locomotion.

32. Eldar E, Cohen JD, Niv Y. The effects of neural gain on attention and learning. Nat Neurosci. 2013;16:1146-53. Correlates locus coeruleus activity with increased functional connectivity in human subjects using pupillometry.

33. Lee SH, Dan Y. Neuromodulation of brain states. Neuron. 2012;76: 209-22.
34. Morgane PJ, Stern WC: The role of serotonin and norepinephrine in sleep-waking activity. Natl Inst Drug Abuse Res Monogr Ser 1975: 37-61.

35. Mouton PR, Pakkenberg B, Gundersen HJ, Price DL. Absolute number and size of pigmented locus coeruleus neurons in young and aged individuals. J Chem Neuroanat. 1994;7:185-90

36. Cohen Z, Molinatti G, Hamel E. Astroglial and vascular interactions of noradrenaline terminals in the rat cerebral cortex. J Cereb Blood Flow Metab. 1997;17:894-904.

37. Devilbiss DM, Waterhouse BD. Norepinephrine exhibits two distinct profiles of action on sensory cortical neuron responses to excitatory synaptic stimuli. Synapse. 2000;37:273-82.

38. Devilbiss DM, Waterhouse BD. Phasic and tonic patterns of locus coeruleus output differentially modulate sensory network function in the awake rat. J Neurophysiol. 2011;105:69-87.

39. Sara SJ. The locus coeruleus and noradrenergic modulation of cognition. Nat Rev Neurosci. 2009;10:211-23.

40. Howells FM, Stein DJ, Russell VA. Synergistic tonic and phasic activity of the locus coeruleus norepinephrine (LC-NE) arousal system is required for optimal attentional performance. Metab Brain Dis. 2012;27:267-74.

41. Constantinople CM, Bruno RM. Effects and mechanisms of wakefulness on local cortical networks. Neuron. 2011;69:1061-8.

42. Schummers J, Yu H, Sur M. Tuned responses of astrocytes and their influence on hemodynamic signals in the visual cortex. Science. 2008;320:1638-43.

43. Iadecola $\mathrm{C}$, Nedergaard $\mathrm{M}$. Glial regulation of the cerebral microvasculature. Nat Neurosci. 2007;10:1369-76.

44. Wang F, Smith NA, Xu Q, Fujita T, Baba A, Matsuda T, et al Astrocytes modulate neural network activity by $\mathrm{Ca}^{(2)+}$-dependent uptake of extracellular K+. Sci Signal. 2012;5.

45. Stout CE, Costantin JL, Naus CC, Charles AC. Intercellular calcium signaling in astrocytes via ATP release through connexin hemichannels. J Biol Chem. 2002;277:10482-8.

46. Szatkowski M, Barbour B, Attwell D. Non-vesicular release of glutamate from glial cells by reversed electrogenic glutamate uptake. Nature. 1990;348:443-6.

47. Kimelberg HK, Goderie SK, Higman S, Pang S, Waniewski RA. Swelling-induced release of glutamate, aspartate, and taurine from astrocyte cultures. J Neurosci. 1990;10:1583-91.

48. Montana V, Malarkey EB, Verderio C, Matteoli M, Parpura V. Vesicular transmitter release from astrocytes. Glia. 2006;54:700-15.

49. Parpura V, Haydon PG. Physiological astrocytic calcium levels stimulate glutamate release to modulate adjacent neurons. Proc Natl Acad Sci U S A. 2000;97:8629-34.

50. Montana V, Ni Y, Sunjara V, Hua X, Parpura V. Vesicular glutamate transporter-dependent glutamate release from astrocytes. J Neurosci. 2004;24:2633-42.

51. Kang J, Kang N, Lovatt D, Torres A, Zhao Z, Lin J, et al. Connexin 43 hemichannels are permeable to ATP. J Neurosci. 2008;28:470211.

52. Kreft M, Potokar M, Stenovec M, Pangrsic T, Zorec R. Regulated exocytosis and vesicle trafficking in astrocytes. Ann N Y Acad Sci. 2009;1152:30-42.

53. Pangrsic T, Potokar M, Stenovec M, Kreft M, Fabbretti E, Nistri A, et al. Exocytotic release of ATP from cultured astrocytes. J Biol Chem. 2007;282:28749-58.

54. Schell MJ, Molliver ME, Snyder SH. D-serine, an endogenous synaptic modulator: localization to astrocytes and glutamatestimulated release. Proc Natl Acad Sci U S A. 1995;92:3948-52.

55. Zonta M, Sebelin A, Gobbo S, Fellin T, Pozzan T, Carmignoto G. Glutamate-mediated cytosolic calcium oscillations regulate a pulsatile prostaglandin release from cultured rat astrocytes. J Physiol. 2003;553:407-14

56. Lee S, Yoon BE, Berglund K, Oh SJ, Park H, Shin HS, et al. Channelmediated tonic GABA release from glia. Science. 2010;330:790-6. 
57. Fujita T, Chen M, Li B, Smith NA, Peng W, Sun W, Toner M, Kress BT, Wang L, Benraiss A, et al.: Neuronal transgene expression in dominant-negative SNARE mice. J Neurosci 2014, 34:16594604.

58. Sun W, McConnell E, Pare JF, Xu Q, Chen M, Peng W, et al. Glutamate-dependent neuroglial calcium signaling differs between young and adult brain. Science. 2013;339:197-200.

59. Thrane AS, Rangroo Thrane V, Zeppenfeld D, Lou N, Xu Q, Nagelhus EA, et al. General anesthesia selectively disrupts astrocyte calcium signaling in the awake mouse cortex. Proc Natl Acad Sci U S A. 2012;109:18974-9.

60. Stickgold R. Sleep-dependent memory consolidation. Nature. 2005;437:1272-8.

61. Gibbs ME, Summers RJ. Role of adrenoceptor subtypes in memory consolidation. Prog Neurobiol. 2002;67:345-91.

62. Sajikumar S, Frey JU. Late-associativity, synaptic tagging, and the role of dopamine during LTP and LTD. Neurobiol Learn Mem. 2004;82:12-25.
63. Tononi G, Cirelli C. Sleep and synaptic homeostasis: a hypothesis. Brain Res Bull. 2003;62:143-50.

64. Vyazovskiy VV, Cirelli C, Pfister-Genskow M, Faraguna U, Tononi G. Molecular and electrophysiological evidence for net synaptic potentiation in wake and depression in sleep. Nat Neurosci. 2008;11:200-8.

65. Cirelli C, Pompeiano M, Tononi G. Neuronal gene expression in the waking state: a role for the locus coeruleus. Science. 1996;274:1211-5.

66. Cirelli C, Tononi G. Locus ceruleus control of state-dependent gene expression. J Neurosci. 2004;24:5410-9.

67. Gibbs ME, Bowser DN. Astrocytic adrenoceptors and learning: alpha1-adrenoceptors. Neurochem Int. 2010;57:404-10.

68. Blanco VM, Stern JE, Filosa JA. Tone-dependent vascular responses to astrocyte-derived signals. Am J Physiol Heart Circ Physiol. 2008;294:H2855-63.

69. Filosa JA, Bonev AD, Straub SV, Meredith AL, Wilkerson MK, Aldrich RW, et al. Local potassium signaling couples neuronal activity to vasodilation in the brain. Nat Neurosci. 2006;9:1397-403. 